\title{
DIAGNOSTICS OF SUPERNUMERARY TEETH IN ORTHODONTIC RELAPSE
}

\author{
Oleksii Bindiuhin \\ Department of Pediatric Dentistry \\ DZ "Dnipropetrovsk Medical Academy of the Ministry of Health of Ukraine" \\ 42 Slobozhansky ave., Dnipro, Ukraine, 49000 \\ dentum76@ukr.net
}

\begin{abstract}
Aim: increasing the effectiveness of diagnosis and further orthodontic-surgical treatment of various clinical forms of hyperdontia (supernumerary teeth) and preventing recurrence with the use of cone-ray computer tomography data in orthodontic practice.

Materials and methods. The clinical case of patient (born in 2004) treatment is presented, with severe hyperdontia and retention of teeth that had little effect on the formation of the general configuration of occlusion. The patient's examination and treatment was carried out with the assistance of clinical, anthropometric and radiological data. The clinical method was to examine the patient and formulate a plan for further orthodontic therapy. The radiological method consisted of data analysis of cefalometry by Bjork, Steiner using a computer program and conducting a survey using a cone-ray computer tomography, followed by the use of these data in diagnosis and orthodontic treatment.

Results. Following the clinical and radiological methods of examination and subsequent orthodontic surgery, the corresponding end results were obtained:

1. The localization and topography of supernumerary teeth as the causes of relapse of pathology with the help of data obtained with the help of CBCT.

2. Radiographic picture reflected on the sagital and axial re-formats of tomo-grams for the diagnosis of supernumerary teeth, is more informative than diagnostics by standard diagnostic protocols.

3. After analysis of the study data, a more meaningful protocol of surgical intervention is prescribed.

4. Implementation of the results of modern diagnostics significantly influenced on the final result of orthodontic treatment.

Conclusions. Thus, in the process of corresponding work installed:

- the availability of computer tomography data is crucial for objective and qualitative diagnosis and treatment of hypertension.

- the general level of diagnostics during the intervention is significantly in-creased.
\end{abstract}

Keywords: hyperodynthia, supernumerary teeth, computed tomography, cephalometric studies, orthodontic-surgical treatment.

\section{Introduction}

In the diagnosis of tooth-jaw anomalies, the frequency of manifestations of the problem of supernumerary teeth is about $0.9 \%$ among other dental anomalies. The frequency of detecting supernumerary teeth by X-ray examination is higher than that obtained in a clinical study. Thus, according to the results of the clinical examination, the level of detected supernumerary teeth was only $0.3 \%$, while simultaneously with the X-ray examination it can reach $4 \%$, which confirms the necessity of using X-ray control of any form of delayed teeth eruption [1, 2].

Usually panoramic radiography is used to detect and diagnose hyperdontia, and thus obtain a two-dimensional, flat, aggregate image of an object. Unlike panoramic tomography using cone-beam computer tomography, the study scans the three-dimensional object completely, which makes it possible to study any part of the jaws in all planes and at any depth [3-6]. The object scan and the future reproduction of the jaw model allows you to determine the spatial position of the teeth, shape, angle of inclination relative to the roots of the adjacent teeth, and most importantly - expands the planning capabilities of removing supernumerary teeth with complex topographic and morphological location $[1,3]$. The use of cone-ray computed tomography originates at the end of the 20th century. The original instrument was first used in the early 1980s of the 20th century by the Mayo Clinic Biodynamics Research Laboratory $[3,7]$ for the study of cardiac and lung functional activity, available as a new diagnostic tool for macho-facial diagnosis, thanks to its work in 1995 Attilio Tacconi and Piero Mozzo at the Quantitative Radiology, Inc. Laboratory of Verona, in Italy. Subsequently, this method was widely presented during the 
following years at international dental congresses. At present, around 32 systems for cone-ray computed tomography are used in the world. The comparative characteristics of the varieties of computed tomography are as follows: the main functional nodes of the scanner act as an $\mathrm{X}-\mathrm{R}$ generator and receiver of the image.

The method of spiral computed tomography (MSCT-multispiral, multi-detector computed tomography), when using the collection of information, passes not in successive layers but the only ray that scans the object in a spiral. When scanning is given the minimum distance between the directions of the helix, the voxel has a characteristic maximum of $0.5 \mathrm{~mm}$. During the study of the skull when used:

- serial convective tomography patient will receive 1000-1500 $\mu \mathrm{Sv}$ (microsurgery);

- spiral tomograph 400-500 $\mu \mathrm{Sv}$;

- cone-ray computer tomography 30-70 $\mu \mathrm{Sv}$ (voltage up to $90 \mathrm{kVp}$ ).

Spiral tomographs work with high levels of current and voltage $(140 \mathrm{kV}, 100 \mathrm{~mA})$, which provides a high beam load on one side, but on the other hand provides the ability to work in several visualization modes: in the lung, in the bone, in the tissue). The radiation load at CBCT is much lower than $50-90 \mu \mathrm{Sv}$ at a current of 3-8 $\mathrm{mA}$, so the processing of the results takes place only in bone window mode, which makes it impossible for a soft tissue diagnosis.

Also, when using CBCT, a beam is used that collects the maximum of information for half the reverse, with additional radial reversal added information that improves the accuracy of the reconstruction. On the other hand, the usual parallel tomography and orthopantomography, where a thin beam is used. In the CBCT technique, the beam is formed in the form of a cone, as with conventional X-ray and with the inversion of the generator around the patient's head, the information arrives at intervals of 30 times per second. Each received image is an output frame [3, 7]. After this, the information goes to the computer, where it is restored in reverse order, thus forming a virtual model, which is converted into a general format DICOM (Digital Imaging Common Medicine), which helps to store information in archival form. In our scientific work, the chilly place when working with computer tomograms is given functions of densitometry method of measuring the optical density of tissues on the Hounsfield scale and obtaining the data displayed in the HU (Hounsfield Unit). Typically, when scanning an object generally accepted, the following imitations of density are in the range ranging from $-1500 \mathrm{HU}$ to $+4000 \mathrm{HU}$ where $-1000 \mathrm{HU}$ is the air density, $0 \mathrm{HU}$ corresponds to the density of water and the density of metal restoration constructions is $+3000 \mathrm{HU}$. It is also proved that there is a possibility of statistical error in the various technical characteristics of the equipment, but the authors recommend to take into account the following indicators of the bone density optical region: for a compact plate 500-1300 HU, for spongy 100$240 \mathrm{HU}$, anterior segment n/850 HU), lateral area N/W $500 \mathrm{HU}$, foremost edge v/o (+500-850 HU), lateral area w/w $(0-500 \mathrm{HU})$. This system is sufficiently flexible and facilitates diagnostic research in planning different stages of orthodontic treatment as a predictor of possible complications. When working with $\mathrm{CBCT}$, the only possible window for research is this "bone window," the image reconstruction uses a modified Feldkamp-Davis-Kress algorithm, a weak voltage (up to $90 \mathrm{kV}$ ) instead of general-medical spiral tomographs where higher voltages (120-125 kV) and high radiation dose, which makes it impossible to unilaterally illuminate information. In orthodontics, the commencement of the use of computed tomography for planning treatment begins in 2005 [2, 5], the first method has been applied in North America. The recent conclusion of the American Association of Orthodontists on this subject establishes the necessary clinical bases for applying the methodology and substantiating its prominent role in the planning of orthodontic therapy in modern conditions: "We must understand the individual clinical situations where there is a value obtained solely as a result of the use of CBCT, but for the sake of planning and conducting simple orthodontic treatment, such a method of research is not needed".

\section{Materials and methods.}

Clinical case of orthodontic-surgical treatment of a patient-boy born in 2002, at the time of treatment, was documented in detail, term of observation from 09.2012 to 06.2014 , turned to the Department of Pediatric Dentistry of the DMA for orthodontic care, he was 14 years old with 
an established orthodontic diagnosis: skeletal class 2, dental alveolar class 1, retention 13, 21, 23 teeth, their vestibular position; dichotomy tooth in the projection of retinal 21 tooth (primary hyperdontia). For the purpose of examination and further treatment, a wide spectrum was involved as data of clinical diagnostics, anthropometry and X-ray examination. The clinical method was the examination of a patient with a tooth-jaw anomaly, including an external examination of the maxillofacial area, and indicators of the oral cavity examination including the conduct of special orthodontic clinical tests to determine the type of facial profile in order to determine the patient's growth tendency. The determination of the width of the dental arc was carried out according to the method by A.Pont and Linder-Harth, and the length of the frontal section of the dental arc method by G. Korkhaus. The measurement of the length of the dentition was carried out by the Nance method using the digital method of studying the models with their previous scan, while the actual length of the dental arch was determined using a digital measurement, which was formed in accordance with the individual form of the dental arc, these indices were compared with the predetermined ones mesiodistal dimensions of crowns of incisors, jaws and premolars, due to which the discrepancy between the expected and actual length of the dental arch was investigated. In our work the Bolton method is also used to determine the proportionality between the mesiodistal dimensions of the lower and upper 6 forearm teeth (incisors and jaws) and to determine the proportionality between the mesiodistal dimensions of the lower and upper 12 permanent teeth-the anterior ratio and the posterior ratio, respectively. The radiological method consisted of a data analysis of cephalometry by Bjork, Steiner, Kim [10] and a cone-ray computed tomography examination with subsequent use of these data in diagnosis and orthodontic treatment $[9,10]$.

\section{Results and their discussion}

Diagnosis of supernumerary teeth placed in the jawbone, which cause to delay the tearing of adjacent teeth, is quite complicated. An effective auxiliary method is a radiographic examination that helps to determine the location of the dichotomy tooth, its morphological features, contacts with surrounding structures and other anatomical features that will play a decisive role in conducting auxiliary surgical intervention in the general plan of orthodontic therapy. In order to more precisely determine the localization of uncut teeth in order to clarify their location (from the vestibular or palatal surfaces of the jaw), and conducting a study of the density of bone tissue by densitometry method, conduct dental cone-ray computer tomography. Its widespread use plays a decisive role in refining the diagnosis in cases of complex orthodontic-surgical treatment of patients. The comparative characteristics of the studies obtained during the panoramic tomography and the cone-ray computer tomography convinces the relevant specialists to lean more towards the greater use of CBCT, in particular in orthodontic practice.

Patient Y. B., 14 years old, asked about the absence of the right maxillary canine, the left central incisor and maxillary canine (Fig. 1).

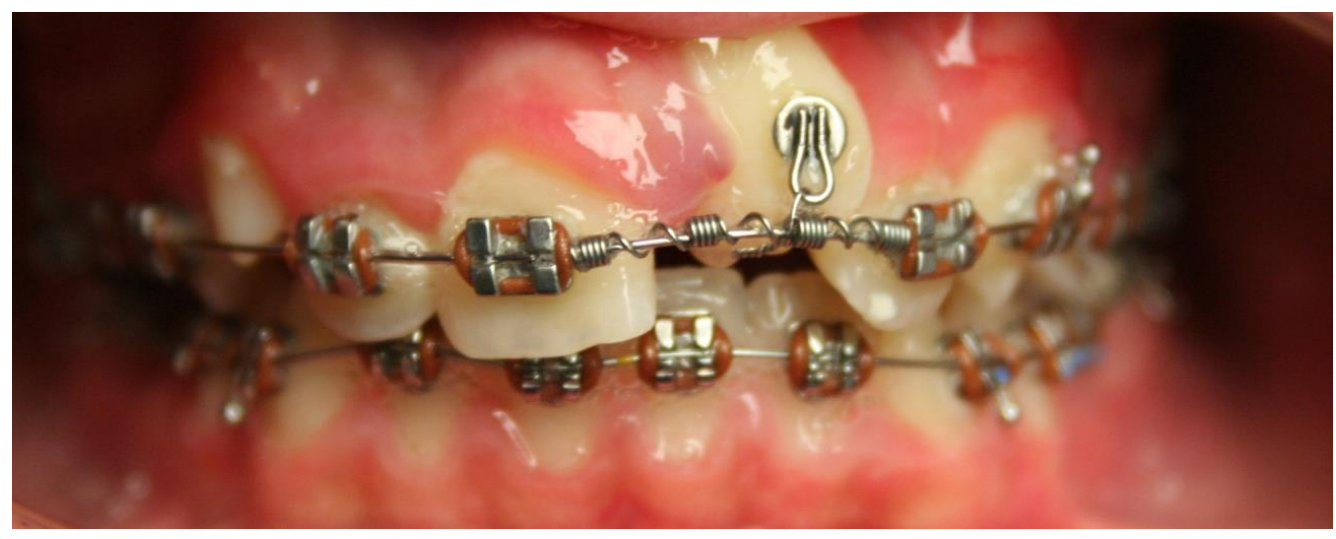

Fig. 1. During the active phase of treatment 
In order to clarify the clinical diagnosis, the data of panoramic tomography (orthopathomography) were used.

A preliminary diagnosis was established: skeletal class 2, dental alveolar class 1 , retention of 13, 21, 23 teeth, their vestibular position; dichotomy tooth in the projection of retinal 21 tooth (primary hyperdontia).

The data of orthopantomography did not give a clear idea about the mutual arrangement of retained and supernumerary teeth, but due to the existence of common stereotypes in society about the health damage from computed tomography at this stage, the parents of the patient gave their written refusal to carry out an appropriate X-ray examination.

In the process of patient examination and orthodontic-surgical treatment with a total duration of 18 months, positive therapeutic changes were made for correction of tooth-jaw anomaly (Fig. 2).

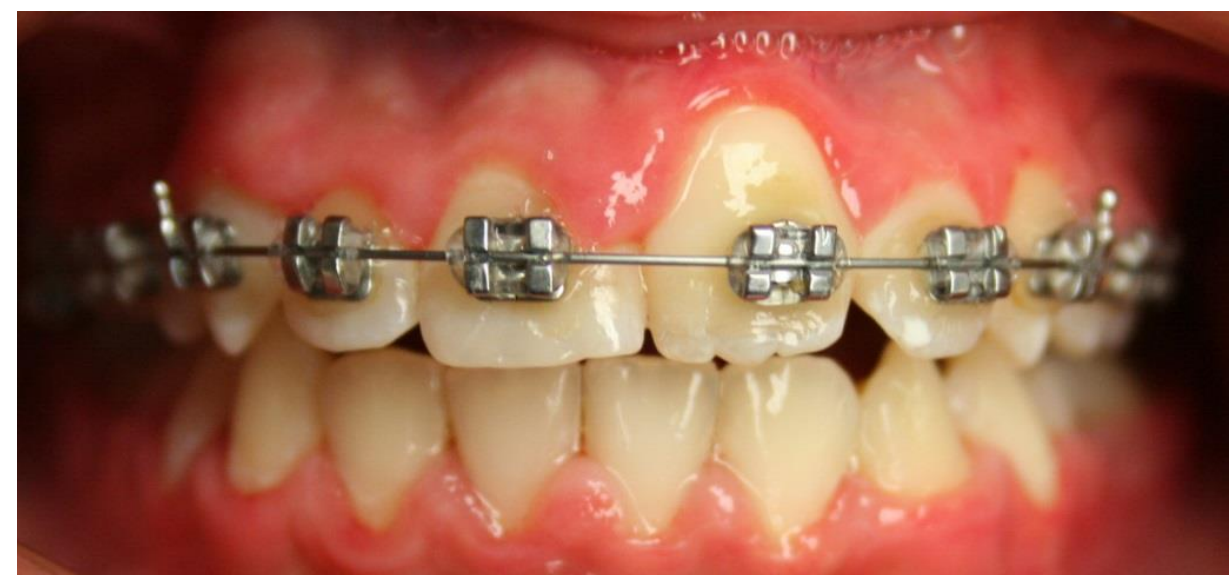

Fig. 2. End of the active phase

That was confirmed by conducting the control X-ray diagnostics using orthopantomography (Fig. 3).

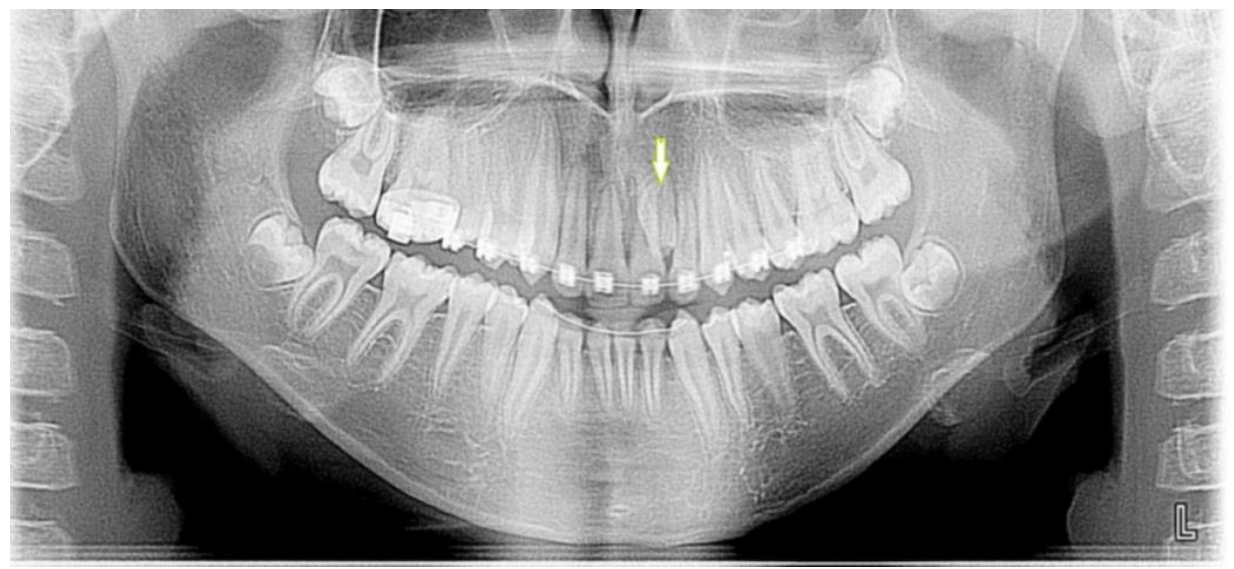

Fig. 3. Orthopantomography after the end of the active phase

The general picture of successful treatment was upset by the fact that there was a discrepancy between soft tissues in the projection of crown 11 and 21 teeth, somewhat the outer position of 21 teeth in a dental arch with a negative thoracic root tooth 21 tooth and the presence of an orthopantomogram of the artifact in the projection of the root of the 21 teeth, which at first glance resembled the presence of another dichotomy tooth with probable localization from the palatine side of the jaw (Fig. 4). 


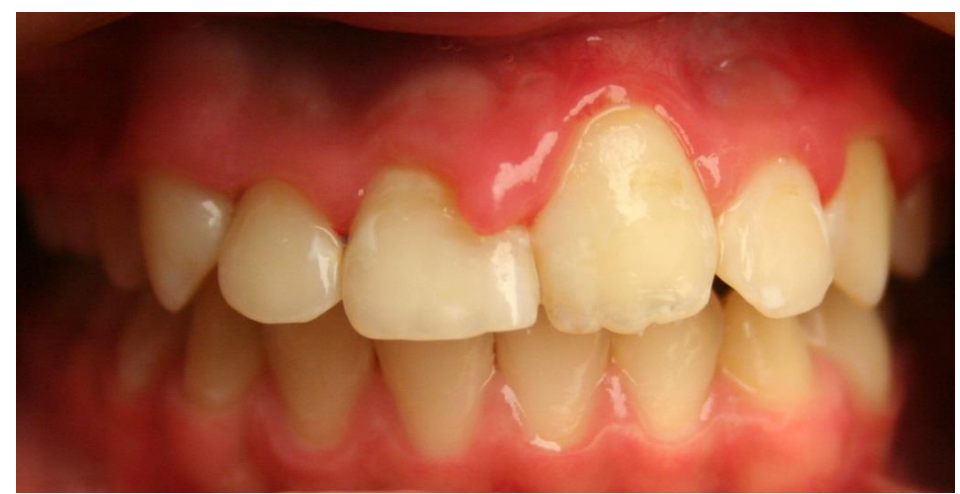

Fig. 4. Visualization of relapse

It was decided to carry out a computer tomography to establish a complete diagnosis that was obtained by the consent of the parents. The results of computer tomography differed significantly from the image on orthopantomogram. There was confirmed the presence of a dichotomy tooth in the projection of the root of the 21 tooth, which probably caused the corresponding position of the tooth and led to a recurrence of overlapping teeth of the upper jaw with systemic pathology of class 2 (Fig. 5, 6).

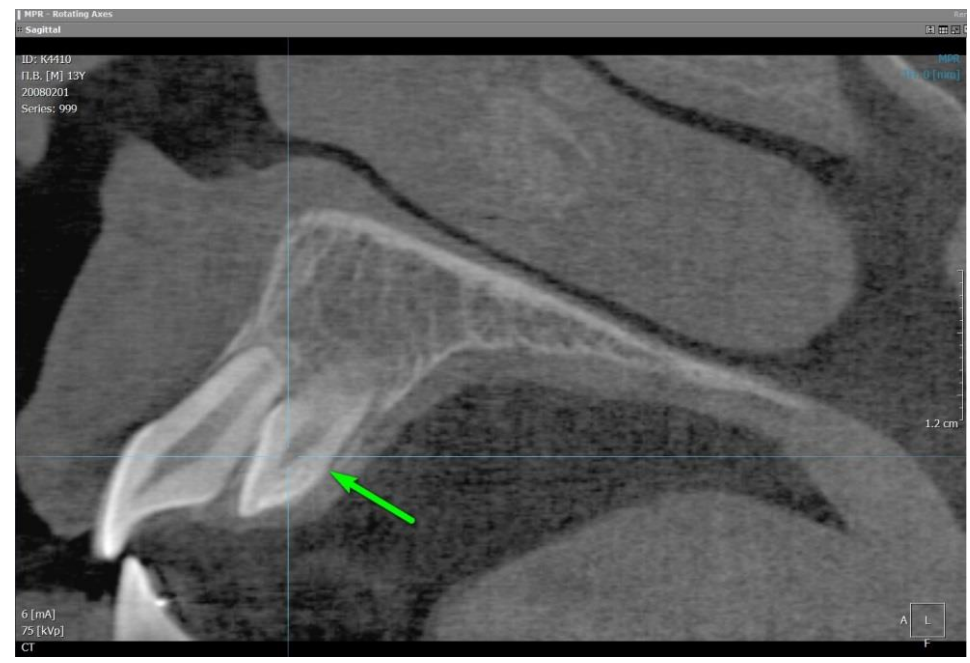

Fig. 5. Sagital reformat

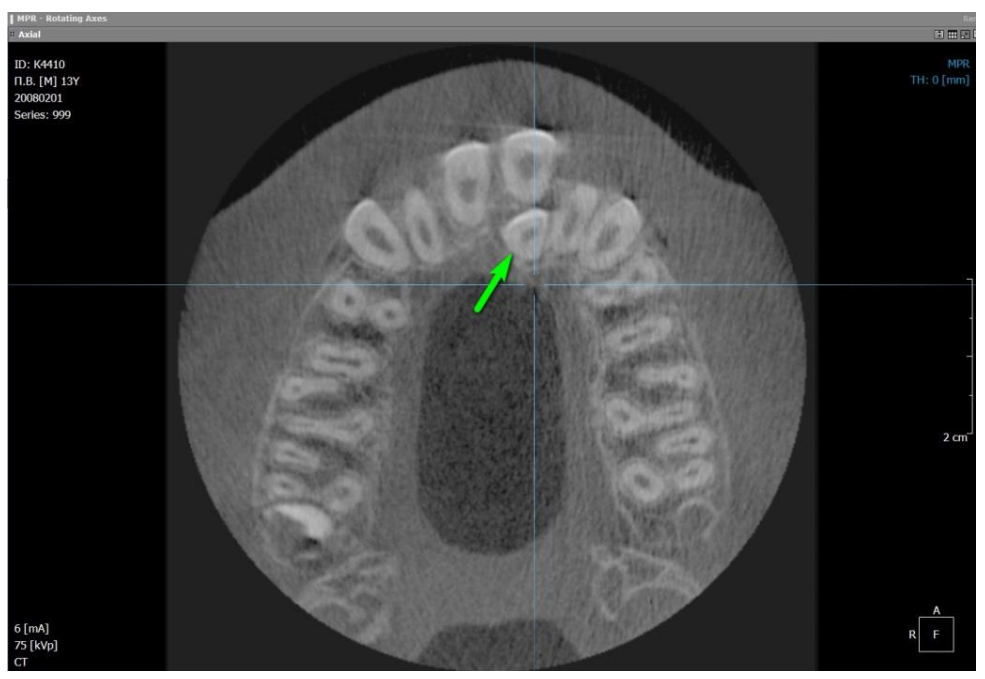

Fig. 6. Axial reformat 
Thus, we state the practical impossibility to establish the exact number of supernumerary teeth in multiple hyperdontia only if the orthopantomography is performed and we can testify to the effectiveness of using tapered-beam tomography for quality diagnostics, refinement of the diagnosis in cases of systemic hyperdontia and multiple retention of permanent teeth in the planning of joint orthodontic-surgical interventions. According to the results of computer tomography, a plan of surgical operation and subsequent re-orthodontic correction was made (Fig. 7).

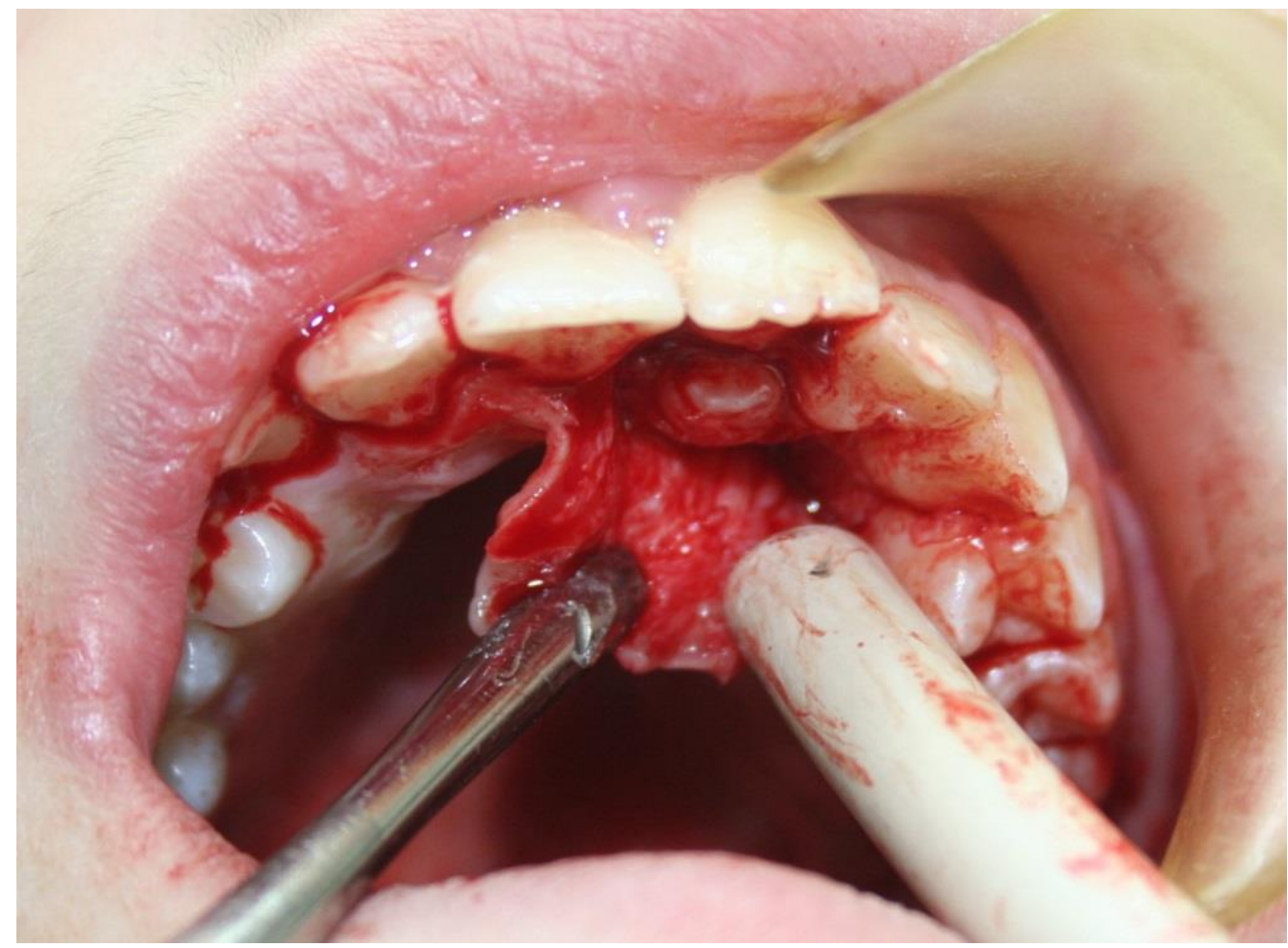

Fig. 7. Surgical stage

\section{Conclusions}

The presence in modern orthodontic practice cases of systemic hyperdontia (cleidocranial dysplasia of the disease of Schaetheuer-Marie-Senton) and more frequent cases of the presence of single supernumerary teeth requires from the orthodontist:

1. A broader and widespread use in the work of the data obtained at the passing of a cone-ray computer tomography.

2. Ability to work out the obtained results for the establishment and refinement of the diagnosis.

3. Planning of future joint orthodontic-surgical interventions in case of relapses of anomaly in systemic orthodontic pathology.

\section{References}

[1] Rohatskin, D. V. (2009). Suchasna kompiuterna tomohrafiia dlia stomatolohii. Implantolohiia Parodontolohiia Osteolohiia, 1, 72-75.

[2] Rogatskin, D. V. (2010). Konusno-luchevaya komp'yuternaya tomografiya. Lviv: GalDent, 148.

[3] Khoroshilkina, F. Ya., Persin, L. S., Okushko-Kalashnikova, V. P. (2005). Ortodontiya: «Profilaktika i lechenie funktsional'nykh, morfologicheskikh i esteticheskikh narusheniı̌ v zubochelyustno-litsevoi oblasti». Moscow, 453.

[4] Korbando, Z.-M., Patts, A. (2009). Khirurgicheskoe i ortodonticheskoe lechenie retinirovannykh zubov. Moscow, 136. 
[5] Khoroshilkina, F. Ya. et. al. (1999). Rukovodstvo po ortodontii. Moscow: Meditsina, 800.

[6] Shul'tts, N. F. (2006). Prakticheskoe rukovodstvo po ortodonticheskoy diagnostike. Lviv: Galdent, 176.

[7] Pauwels, R., Beinsberger, J., Collaert, B., Theodorakou, C., Rogers, J., Walker, A. et. al. (2012). Effective dose range for dental cone beam computed tomography scanners. European Journal of Radiology, 81 (2), 267-271. doi: http://doi.org/10.1016/j.ejrad.2010.11.028

[8] Mavragani, M., Boe, O. E., Wisth, P. J., Selvig, K. A. (2002). Changes in root length during orthodontic treatment: advantages for immature teeth. The European Journal of Orthodontics, 24 (1), 91-97. doi: http://doi.org/10.1093/ejo/24.1.91

[9] Fuhrmann, R. (1996). Dreidimensionale Interpretation parodontaler Läsionen und Remodellationen im Verlauf orthodontischer Behandlungen. Journal of Orofacial Orthopedics/Fortschritte Der Kieferorthopädie, 57 (4), 224-237. doi: http://doi.org/10.1007/bf02190235

[10] Jeong, K. (2016). Il Meaw Well. Publishing Seoul, 656. 